\title{
Human Synthesis and Scene Compositing
}

\author{
Mihai Zanfir, ${ }^{3,1}$ Elisabeta Oneata, ${ }^{3,1}$ Alin-Ionut Popa, ${ }^{3,1}$ Andrei Zanfir, ${ }^{1,3}$ Cristian Sminchisescu ${ }^{1,2}$ \\ ${ }^{1}$ Google Research ${ }^{2}$ Department of Mathematics, Faculty of Engineering, Lund University \\ ${ }^{3}$ Institute of Mathematics of the Romanian Academy \\ \{mihai.zanfir, elisabeta.oneata, alin.popa, andrei.zanfir\}@imar.ro, sminchisescu@google.com
}

\begin{abstract}
Generating good quality and geometrically plausible synthetic images of humans with the ability to control appearance, pose and shape parameters, has become increasingly important for a variety of tasks ranging from photo editing, fashion virtual try-on, to special effects and image compression. In this paper, we propose a HUSC (HUman Synthesis and Scene Compositing) framework for the realistic synthesis of humans with different appearance, in novel poses and scenes. Central to our formulation is $3 \mathrm{~d}$ reasoning for both people and scenes, in order to produce realistic collages, by correctly modeling perspective effects and occlusion, by taking into account scene semantics and by adequately handling relative scales. Conceptually our framework consists of three components: (1) a human image synthesis model with controllable pose and appearance, based on a parametric representation, (2) a person insertion procedure that leverages the geometry and semantics of the $3 d$ scene, and (3) an appearance compositing process to create a seamless blending between the colors of the scene and the generated human image, and avoid visual artifacts. The performance of our framework is supported by both qualitative and quantitative results, in particular state-of-the art synthesis scores for the DeepFashion dataset.
\end{abstract}

\section{Introduction}

Generating photorealistic synthetic images of humans, with the ability to control their shape and pose parameters, and the scene background is of great importance for end-user applications such as in photo-editing or fashion virtual tryon, and for a variety of data-hungry human sensing tasks, where accurate ground truth would be very difficult if not impossible to obtain (e.g. the $3 \mathrm{~d}$ pose and shape of a dressed person photographed outdoors).

One way to approach the problem would be to design human models and $3 \mathrm{~d}$ environments using computer graphics. While the degree of realism increased dramatically in narrow domains like the movie industry, with results that pass the visual Turing test, such synthetic graphics productions require considerable amount of highly qualified manual work, are expensive, and consequently do not scale. In

Copyright (c) 2020, Association for the Advancement of Artificial Intelligence (www.aaai.org). All rights reserved. contrast, other approaches avoid the $3 \mathrm{~d}$ modeling pipeline altogether, aiming to achieve realism by directly manipulating images and by training using large-scale datasets. While this is cheap and attractive, offering the advantage of producing outputs with close to real statistics, they are not nearly as controllable as the $3 \mathrm{~d}$ graphics ones, and results can be geometrically inconsistent and often unpredictable.

In this work we attempt to combine the relatively accessible methodology in both domains and propose a framework that is able to realistically synthesize a photograph of a person, in any given pose and shape, and blend it veridically with a new scene, while obeying $3 \mathrm{~d}$ geometry and appearance statistics. An overview is given in fig. 1.

For the first part of human synthesis, given a source image of a person and a different target pose (or more generally, a target $3 \mathrm{~d}$ body mesh), we want to generate a realistic image of the person synthesized into the new pose. We would like that all the elements included in the person's source layout (either clothing, accessories or body parts) to be preserved or plausibly extended in the synthesis. We propose to learn a dense displacement field, that leverages $3 \mathrm{~d}$ geometry and semantic segmentation (e.g. a blouse moves differently than a hat; the leg moves differently than the head). This module produces a correction to an initial body displacement field and is trained jointly with the synthesis model within a single end-to-end architecture.

Finally, given an image of a person (real or synthesized) and of a background scene, we want to generate a good quality composite of the two. We argue that a realistic synthesis should consider the physical properties of the scene and of the human body, and also compensate for the different appearance statistics. With that in mind, we propose to blend the foreground image with the background image, at two levels: geometry and appearance. At the geometric level, we want the foreground person, with its associated $3 \mathrm{~d}$ body model, to respect the $3 \mathrm{~d}$ space and scale constraints of the scene, be visible according to the scene depth ordering, and be placed on a plausible support surface (e.g. floor). At the appearance level, we would like that the two sources blend naturally together, without the undesirable cut-andpaste look. To summarize, our contributions are as follows: (a) a realistic human appearance translation task, with state- 


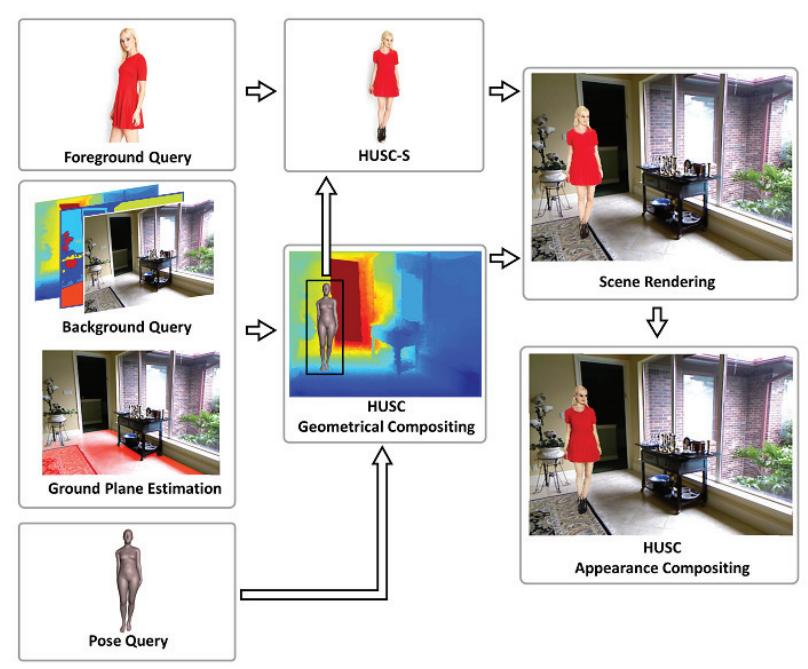

Figure 1: Overview of our full pipeline HUSC. Inputs are a foreground image of a person, a background scene with its associated depth map and semantic labeling, and a target $3 \mathrm{~d}$ body model. First, we perform ground plane estimation of the background scene. Within the Geometrical Compositing stage, we sample a valid $3 \mathrm{~d}$ location for the target body and perform the associated viewpoint transformation and alignment with the supporting plane normal. The newly updated target body shape, together with the input image encoding the desired appearance, are passed to the human synthesis network, HUSC-S. The resulting synthesized foreground image is rendered in the background scene, by properly accounting for depth ordering constraints. Finally, its appearance is altered by our learned Appearance Compositing network in order to produce the final result.

of-the-art results, (b) a realistic data augmentation procedure, which allows for the synthesis of complex scenes containing humans, with available pseudo-ground-truth labels such as: pose, shape, segmentation and depth.

\section{Related Work}

Human Image Synthesis. An important body of work in the literature is dedicated to image synthesis (Goodfellow et al. 2014; Nguyen et al. 2017; Isola et al. 2017; Yi et al. 2017; Lee et al. 2018; Wang et al. 2018; Luan et al. 2017; Johnson, Alahi, and Fei-Fei 2016), and more specifically to the task of synthesizing photo-realistic images of humans (Zanfir, Popa, and Sminchisescu 2018; Ma et al. 2017; Han et al. 2018; Ma et al. 2018; Siarohin et al. 2018; Lassner, Pons-Moll, and Gehler 2017; Grigorev et al. 2018; Li, Huang, and Loy 2019). Among these, a significant proportion - our work included - have focused on synthesizing humans given a condition image and a desired target pose. In (Esser, Sutter, and Ommer 2018) the authors propose a variational U-Net for conditional image generation. Their method synthesizes images of humans based on $2 \mathrm{~d}$ pose information and a latent appearance representation learned with a variational auto-encoder. We leverage a richer shape and position representation in the form of a $3 \mathrm{~d}$ body model and learn a dense correspondence field between the pose and shape of the source person and that of the target. This motion field is extended beyond body regions to clothing and hair. (Dong et al. 2018) learns a transformation grid based on affine and thin-plate spline embedding, which is used to warp the condition image in the desired target position. In contrast, our learned displacement field allows for dense arbitrary transformations. Our work relates to (Siarohin et al. 2018), through the use of deformable skip connections for warping the features maps of the conditioning image, in the location of the desired target pose. However, there are two major differences between our method and the DSCF net proposed in (Siarohin et al. 2018): i) In DSCF net, the image features are warped with an affine transformation obtained through an optimization step both at training and at testing time. ii) The deformable skip connection in DSCF net transform feature-maps, that correspond to coarse $2 \mathrm{~d}$ body joint activations, while our learned displacement field is densely computed over the entire person layout (body and clothing). Different from (Zanfir, Popa, and Sminchisescu 2018)(HAT), we learn end-to-end the dense correspondence field, coupling it together with the synthesis part of our network. HAT operates in two different stages: one that synthesizes color only on the target body shape, and one that tries to further complete the color on the outside regions. This latter stage is completely detached from the initial source image and is guided only by a target clothing segmentation.

General data augmentation through image synthesis. Combining different sources of synthetic data with real data, and then generating a realistic composition of the both, has been successfully applied to various tasks, such as semisupervised foreground-background segmentation (Remez, Huang, and Brown 2018; Alhaija et al. 2018; Dwibedi, Misra, and Hebert 2017), object detection (Dvornik, Mairal, and Schmid 2018; Dwibedi, Misra, and Hebert 2017) or $3 \mathrm{~d}$ object pose estimation (Alhaija et al. 2018). The two cut-and-paste methods (Dwibedi, Misra, and Hebert 2017; Remez, Huang, and Brown 2018) use simple blending techniques, and only for the foreground object, while we propose to learn a blending for both the background and foreground, accordingly. Note that while (Alhaija et al. 2018) and (Dwibedi, Misra, and Hebert 2017) take the $3 \mathrm{~d}$ geometry of the scene into account, they only consider $3 \mathrm{~d}$ rigid objects.

Human Synthesis on Backgrounds. Most of the previous works focus solely on the human appearance, and are not concerned with a natural blending within a background image. There are some works that consider synthesizing the entire scene, such as (Varol et al. 2017), where textured human body models are overlaid over random backgrounds. This method does not consider the semantic and the $3 \mathrm{~d}$ structure of the scene, hence, the human model is often placed in unnatural locations. Moreover, the model's dimensions and illumination do not match the ones of the surrounding environment. Another relevant work is the one of (Balakrishnan et al. 2018), that takes as input an image containing a human and its desired $2 \mathrm{~d}$ skeleton configuration. The method 

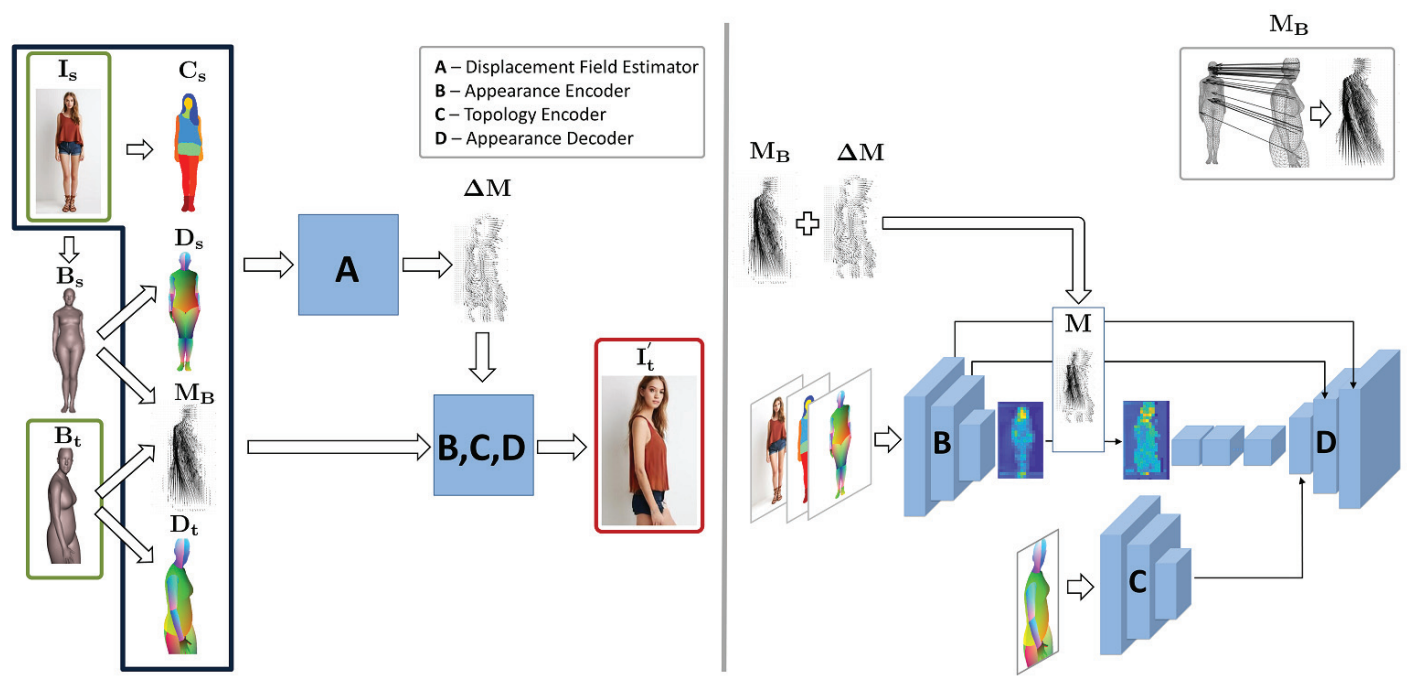

Figure 2: (Left) Overview of HUSC-S. Our pipeline receives as input (shown inside green boxes) a source monocular image of a person, $I_{s}$, and a desired target $3 \mathrm{~d}$ body mesh, $B_{t}$. We estimate for $I_{s}$ a $3 \mathrm{~d}$ body mesh, $B_{s}$, and a clothing segmentation, $C_{s}$. From $B_{s}$ and $B_{t}$, we can compute a dense body displacement field $M_{B}$, and also their respective dense pose representations, $D_{s}$ and $D_{t}$. The displacement field estimator (A), on the top branch, receives as input all the available information, for both source and target. It outputs an update $\Delta M$ that is added to $M_{B}$ to produce the final displacement field, $M$. (Right) Detailed view of HUSC-S. The appearance encoder (B) receives as input only the information pertaining to the source. It produces feature maps at different resolutions that are all displaced according to $M$. We show an example of such a transformation at the lowest resolution. The topology encoder (C) operates on the target dense pose, $D_{t}$, and is input to the decoder $\mathbf{D}$ to help guide the synthesis, alongside all the feature maps coming from $\mathbf{B}$. The output of our network is the final synthesized image $I_{t}^{\prime}$ (shown inside the red box, left).

changes the position of the human to the given pose configuration, while retaining the background scene and inpainting the missing areas.

\section{Human Synthesis}

Overview. Given a source RGB image $I_{s}$ of a clothed person, our goal is to realistically synthesize the same person in a given novel pose, in our case represented by a $3 \mathrm{~d}$ body model, $B_{t}$. Different from previous works that rely mostly on $2 \mathrm{~d}$ joints to model the pose transformation, one of our key contributions lies in incorporating richer representations, such as $3 \mathrm{~d}$ body shape and pose which improves the predictions in complicated cases - e.g. determining how the position/occlusion of the body and clothing/hair change with the motion/articulation of the person. Moreover, a 3d body model is required to plausibly place the person in the scene and allows us to have full control over $3 \mathrm{~d}$ body shape, pose, and the scene location where the synthesized person is inserted.

Our human synthesis model is a conditional - GAN (Wang et al. 2018) that consists of a generator $\mathcal{G}$ and a discriminator $\mathcal{D}$. Given a source image $I_{s}$ and a condition pose $B_{t}$, the task of the generator is to produce an output image $I_{t}^{\prime}=\mathcal{G}\left(I_{s}\right)$ of the person in $I_{s}$ having the body pose $B_{t}$. The discriminator's objective is to distinguish between real and generated images while the generator's task is to produce fake images which are able to fool the discriminator.

Starting from an encoder-decoder architecture, our key contribution is the addition of a novel Displacement Field Estimator in the generator, that learns to displace features from the encoder, prior to being decoded. Our modified architecture also relies on the semantic segmentation (i.e. clothing and body parts) of the source image, $C_{s}$, and on an estimated $3 \mathrm{~d}$ body model of the source, $B_{s}$. An overview of our generator can be seen in fig. 2. We use a multiscale discriminator as in (Wang et al. 2018).

3d Body Model Estimation. For estimating the 3d body model of an image, we use the SMPL model (Loper et al. 2015) and follow a similar procedure to (Zanfir, Marinoiu, and Sminchisescu 2018), but instead apply the method of (Zanfir et al. 2018) for 3d pose estimation. The SMPL model is a parametric $3 \mathrm{~d}$ human mesh, controlled by pose parameters $\boldsymbol{\theta} \in \mathbb{R}^{24 \times 3}$ and shape parameters $\boldsymbol{\beta} \in \mathbb{R}^{10}$, that generate vertices $\mathbf{V}(\boldsymbol{\theta}, \boldsymbol{\beta}) \in \mathbb{R}^{6890 \times 3}$. We fit this model to predicted $2 \mathrm{~d}$ keypoints and semantic segmentation, under a fullperspective model with fixed camera intrinsics. We refer to the tuple $(\boldsymbol{\theta}, \boldsymbol{\beta}, \mathbf{T})$ as a model $B$, where $\mathbf{T}$ is the inferred camera-space translation.

Dense Body Correspondences. Given two body meshes (i.e. a source and a target) we can compute a $3 \mathrm{~d}$ displacement field $M_{B}^{3 d}$ of the visible surface points on the source $\mathbf{V}_{\mathbf{s}}\left(\boldsymbol{\theta}_{s}, \boldsymbol{\beta}_{s}\right)$, to the target surface points $\mathbf{V}_{\mathbf{t}}\left(\boldsymbol{\theta}_{t}, \boldsymbol{\beta}_{t}\right)$ (see fig. 2 , top right corner).

$$
M_{B}^{3 d}=\mathbf{V}_{\mathbf{s}}\left(\boldsymbol{\theta}_{s}, \boldsymbol{\beta}_{s}\right)-\mathbf{V}_{\mathbf{t}}\left(\boldsymbol{\theta}_{t}, \boldsymbol{\beta}_{t}\right)
$$


The displacement field is projected in $2 \mathrm{~d}$, and encoded onto the target shape, representing the offset from where information is being transferred. We will refer to this final $2 \mathrm{~d}$ displacement field as $M_{B}=\mathcal{P}\left(M_{B}^{3 d}\right)$.

Topological Representation. From a 3d body mesh we obtain a 2 d dense pose (Alp Guler, Neverova, and Kokkinos 2018) representation for both the source, $D_{s}$, and the target, $D_{t}$. The dense pose representation encodes at pixel level, for the visible $3 \mathrm{~d}$ body points, the semantic body part it corresponds to, alongside the $2 \mathrm{~d}$ coordinates in the local body part reference system. Each $3 \mathrm{~d}$ body point will therefore have a unique associated value using this encoding scheme.

Image Synthesis Network. Our image synthesis network generator (HUSC-S) has two novel computational units: a Displacement Field Estimator, and a Topology Encoder. The former learns an update, $\Delta M$, for the displacement field $M_{B}$, which is meant to either correct the warping induced by an erroneous fitting, or capture the motion of structures that fall outside the fitted model (e.g. hair, clothing). The final displacement field is given by $M=\Delta M+M_{B}$, and is used to place source features at the correct spatial location in the target image, to facilitate decoding and thus, synthesis. This update step is computed on geometric and semantic features, namely: $I_{s}, D_{s}, D_{t}, C_{s}$ and $M_{B}$.

An Appearance Encoder module extracts features $A_{s}$ based on the RGB image $I_{s}$, a pre-computed clothing segmentation $C_{s}$ (using (Gong et al. 2018)) and the source dense pose, $D_{s}$. The updated displacement field $M$ is used to warp the appearance features $A_{s}$ and produce warped image features $A_{t}^{\prime}$. These are then passed through a series of residual blocks. The result is concatenated with an encoding of $D_{t}$, produced by the Topology Encoder, and then passed to an Appearance Decoder to generate the synthesized image, $I_{t}^{\prime}$. This additional information is useful to the decoding layers, since the underlying body-part topology is exposed to them. We add deformable (i.e. warped by our estimated displacement field) skip connections between the Appearance Encoder and the Appearance Decoder, to propagate lower level features directly to the decoding phase.

Training. At training time, we are given pairs of images $\left\{\left(I_{s}, I_{t}\right)\right\}$ which are used to learn both the parameters of the discriminator, $\theta_{d}$, and those of the generator, $\theta_{g}$, using a combined $L 1$ color loss, a perceptual VGG loss (Johnson, Alahi, and Fei-Fei 2016) and a discriminator GAN loss.

$$
\begin{gathered}
\mathcal{L}=\mathcal{L}_{G A N}+\lambda \mathcal{L}_{L 1}+\gamma \mathcal{L}_{V G G} \\
\mathcal{L}_{G A N}=\mathbb{E}_{\left(I_{s}, I_{t}\right)}\left[\log \mathcal{D}\left(I_{s}, I_{t}\right)\right]+ \\
\mathbb{E}_{I_{s}}\left[\log \left(1-\mathcal{D}\left(I_{s}, \mathcal{G}\left(I_{s}\right)\right)\right]\right. \\
\mathcal{L}_{L 1}=\sum_{(u, v)}\left\|I_{t}(u, v)-I_{t}^{\prime}(u, v)\right\|_{1} \\
\mathcal{L}_{V G G}=\sum_{i=1}^{N} \frac{1}{N_{i}}\left\|V G G^{(i)}\left(I_{t}\right)-V G G^{(i)}\left(I_{t}^{\prime}\right)\right\|_{1}
\end{gathered}
$$

$N$ represents the number of layers considered from the VGG network, $N_{i}$ is the dimension of the output features and $V G G^{(i)}(I)$ are the activations of the $i^{t h}$ layer on image $I$.

\section{Human and Scene Compositing}

In image compositing, we are interested in combining two visual elements from different sources into a single image, creating the impression that they are actually part of the same scene. In order to create this effect, the addition of the person in the scene should respect the physical laws of the real world, and the appearance statistics of the person (given by illumination, contrast, saturation, resolution) should match those of the scene. To meet our goals, we consider that we have a background image with associated depth and semantic information, and a foreground image of a person (synthesized or real) with an estimated $3 \mathrm{~d}$ body model (see fig. 1 for an overview).

\section{Geometrical Compositing}

We are interested in generating images that respect real world physical constraints, such as: people are (usually) sitting on a ground plane, do not penetrate objects in the scene and have plausible body proportions considering the scale of the environment. In order to estimate a ground plane, we select all $3 \mathrm{~d}$ points that correspond to the semantic classes associated with a floor plane surface. We fit a plane using a least-squares solution, combined with a RANSAC approach to ensure robustness to outliers. This is necessary to mitigate the noise in the depth signal.

In order to choose a physically plausible position for the body model in the scene, we sample $3 \mathrm{~d}$ floor locations on a regular grid. To make the model perpendicular to the plane, we compute a rotation matrix that aligns the "up" vector of the model to the plane normal, and multiply it with the original rotation matrix. Also, due the computed camera-space translation, the relative camera orientation will change. Adjusting for both the rotation induced by the plane normal alignment, and the camera point-of-view, involves a rotation of the original SMPL model. Next, we test whether the mesh model collides with other $3 d$ points in the scene. We approximate the body model with simple $3 \mathrm{~d}$ primitives and check for intersections. If no collisions are detected, we can then place the foreground image. Notice that this is not at the reach of other methods, as they do not have the means to correct their foreground hypotheses based on $3 \mathrm{~d}$ transformations.

In order to correct the appearance change, associated with these transformations, we run our HUSC-S component for the foreground image and its updated corresponding $3 \mathrm{~d}$ model. We select the color of a pixel according to the relative depth ordering of $3 \mathrm{~d}$ points, belonging to both the model and the scene (e.g if the model is behind a desk, some of its body parts will be occluded). An important property of this procedure is its scalability: none of the components requires human intervention, as the whole pipeline is fully automated. 


\section{Appearance Compositing}

In order to create a natural composition of foreground and background image pairs, we build on a methodology originally proposed in (Tsai et al. 2017) for generating realistic composites of arbitrary object categories over backgrounds. (see fig. 3 for an overview). We learn an adjustment of the color and boundaries of a human composite such that it blends naturally (in a statistical, learned sense) in the scene. For training we use images with humans from COCO. We alter image statistics inside the human silhouette so that it looks unnatural, thus simulating a silhouette pasted on a different background. The network learns to recreate the original image.

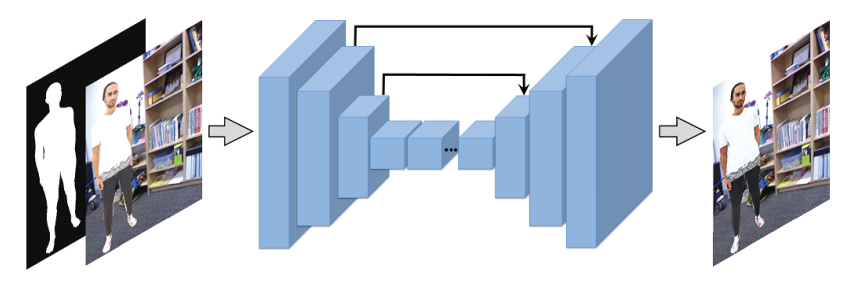

Figure 3: Our Appearance Compositing architecture is represented by an encoder-decoder network inspired by the generator used in (Wang et al. 2018). It takes as input the geometrical composited image and a figure ground segmentation of the person, and corrects his appearance in order to naturally match the scene of insertion.

We make a series of adjustments to the methodology proposed in (Tsai et al. 2017). First, we restrict the problem to generating realistic composites of only humans and backgrounds. We build our model based on a different, more recent network architecture (Wang et al. 2018) and we drop the scene parsing task, since our compositing problem is defined on a specific category (humans). The network's input is a concatenation of the modified image and the binary figureground mask (see fig. 3). We add skip connections between the encoder and decoder, such that the network can easily access background features in the decoding stage. We also remove the batch normalization layers, so that the original image statistics are preserved. We train the network by using a combined $L 1$ color loss, a perceptual VGG loss (Johnson, Alahi, and Fei-Fei 2016) and a discriminator GAN loss.

\section{Experimental Results}

Evaluation metrics. We use several metrics to test the quality of our generated images: the Learned Perceptual Image Patch Similarity metric (LPIPS) (Zhang et al. 2018), the Inception Score (IS) (Salimans et al. 2016) and the Structural Similarity Index (SSIM) (Wang et al. 2004).

\section{Human Synthesis}

Datasets. We train our model on the DeepFashion (Inshop Clothes Retrieval Benchmark) (Liu et al. 2016) dataset, which contains 52,712 in-shop clothes images and around 200,000 cross-pose/scale pairs. We use the train/test split provided by (Siarohin et al. 2018), containing 101, 268 train and 8,616 test pairs of the same person in two different poses.

Quantitative Evaluation. In table 1 we provide quantitative results of HUSC-S on the DeepFashion dataset and compare them with previous works. In addition, we perform an ablation study to show the impact of the learned displacement field in the quality of the generated images. Our first baseline (HUSC-S w/o displacement field) is a simplified version that does not use a displacement field and its associated encoder. The source image, $I_{s}$, source dense pose $D_{s}$, source clothing segmentation $C_{s}$, and target dense pose $D_{t}$ are concatenated and passed directly to the Appearance Encoder. As can be seen in table 1, this is a strong baseline, achieving competitive results with previous works under the IS metric, and state-of-the-art results under SSIM. Its strength lies in leveraging more complex pose and shape information in the form of dense pose (previously only used by DPT (Neverova, Alp Guler, and Kokkinos 2018)). In the second baseline (HUSC-S w/ fixed body displacement field), we compute the body displacement field between the $3 \mathrm{~d}$ body model of the source image and that of the target image, and use it to warp the encoded appearance features of the source. Even if this displacement field is fixed and limited only to body regions, it still improves the results over the first baseline. In the third baseline, we use a dedicated Displacement Field Estimator (see fig. 2) to learn a correction of the initial body displacement, that is extended to clothes and hair. The appearance features of the source image are warped with the corrected motion field, right before the residual blocks computation. By doing so, we obtain the largest performance increase, as well as state-of-the-art results on both IS and SSIM scores. This shows the positive impact that the learned displacement field has on the quality of the generated images. The full method (HUSC-S full) adds two elements: i) the deformable skip connections between the encoder and the decoder for faster convergence and ii) the target dense pose in the decoding phase through the Topology Encoder, such that information on the position in which the person should be synthesized is explicitly available.

\begin{tabular}{|l|c|c|}
\hline Methods & IS $\uparrow$ & SSIM $\uparrow$ \\
\hline \hline pix2pix(Isola et al. 2017) & 3.249 & 0.692 \\
\hline PG2(Ma et al. 2017) & 3.090 & 0.762 \\
\hline DSCF(Siarohin et al. 2018) & 3.351 & 0.756 \\
\hline UPIS(Pumarola et al. 2018) & 2.970 & 0.747 \\
\hline BodyROI7(Ma et al. 2018) & 3.228 & 0.614 \\
\hline AUNET(Esser, Sutter, and Ommer 2018) & 3.087 & 0.786 \\
\hline DPT(Neverova, Alp Guler, and Kokkinos 2018) & 3.61 & 0.785 \\
\hline SGW-GAN(Dong et al. 2018) & 3.446 & 0.793 \\
\hline DIAF (Li, Huang, and Loy 2019) & 3.338 & 0.778 \\
\hline \hline HUSC-S w/o displacement field & 3.3876 & 0.8035 \\
\hline HUSC-S w/ fixed body displacement field & 3.4541 & 0.8016 \\
\hline HUSC-S w/ learned displacement field & 3.6343 & 0.8049 \\
\hline HUSC-S full & $\mathbf{3 . 6 9 5 0}$ & $\mathbf{0 . 8 1 3 5}$ \\
\hline
\end{tabular}

Table 1: Evaluation on the DeepFashion dataset. We perform several ablation studies, showing that learning the displacement field, compared to no or fixed body displacement, performs better in terms of both IS and SSIM scores. Our full method obtains state-of-the art results. 

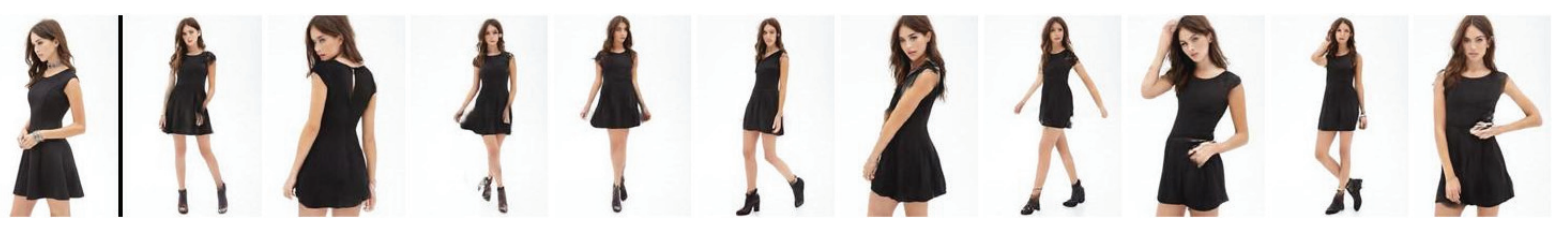

Figure 4: Appearance transfer results of a single RGB image into various poses. The first image represents the source, while the others are obtained by synthesizing that person in different poses.

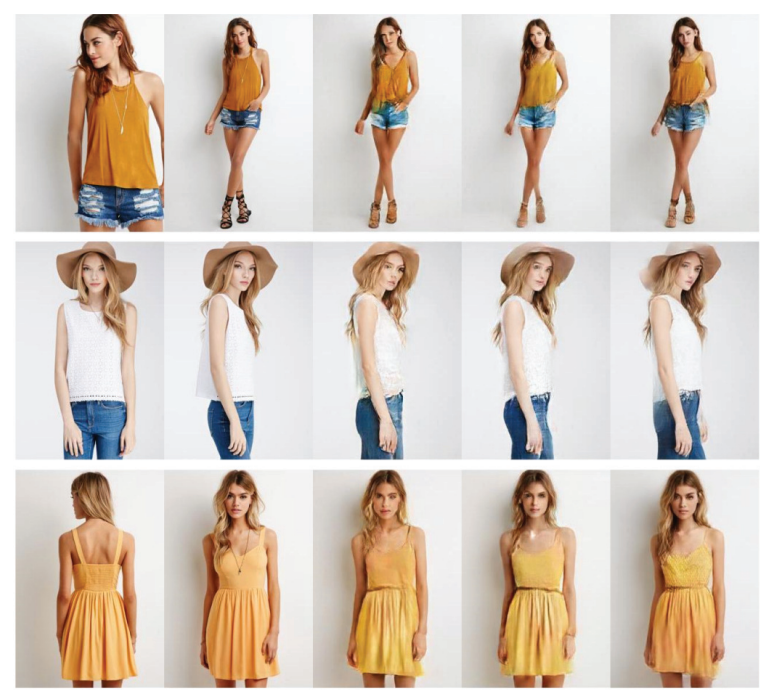

Figure 5: Sample results on the DeepFashion dataset. From left to right: source image, target image, HUSC-S w/ fixed body displacement field, HUSC-S w/ learned displacement field, HUSC-S full. Notice the superior quality of the images synthesized with learned displacement field.

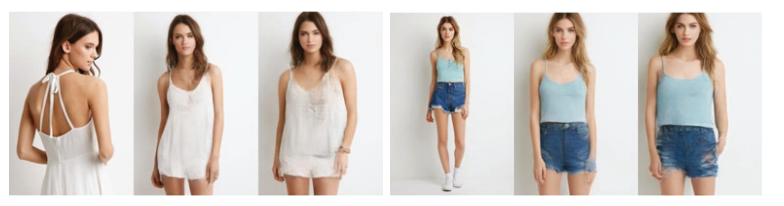

Figure 6: Human synthesis with varying shape parameters. (Left) Source image. (Center) Synthesized image with same shape parameters as the source. (Right) Synthesized image with larger shape parameters than the source.

Qualitative Evaluation. A visual comparison of the baselines and the full method can be seen in fig. 5. The first column represents the source image, the second column is the ground truth target image, while the last three columns are outputs of the following methods: HUSC-S w/ fixed body displacement field, HUSC-S w/ learned displacement field and HUSC-S full. Notice the superior quality of the images synthesized with learned displacement (columns 4 and 5) in terms of i) pose: the order in which the legs overlap in the first image, ii) clothing details: the folds of the dress in the third row look more realistic when using learned motion field, iii) color/texture preserving: the color in the first row
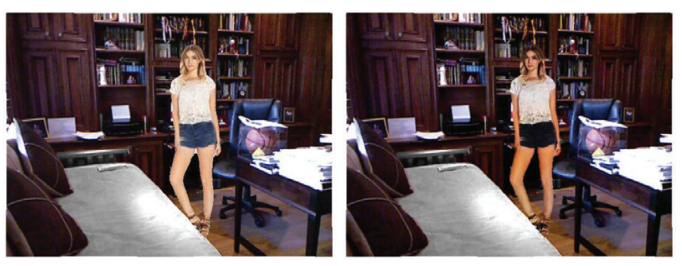

Figure 7: Before and after Appearance Compositing. We learn an adjustment of the color and boundaries of a human composite such that it blends naturally (in a statistical, learned sense) in the scene.

and the hat in the second row better resemble those of source image and iv) face details: in all cases the face of the synthesized person is sharper and better resembles the source person when using the full method. Our method can synthesize high-quality images of persons in a wide range of poses and with a large variety of clothing types, both loose and closely fitted to the body. Moreover, HUSC-S allows altering the underlying $3 \mathrm{~d}$ pose and shape of the desired target person, while correspondingly adjusting the appearance. Please see fig. 6 for examples of body proportions variations, and fig. 4 for synthesized images of the same person in various poses.

\section{Appearance and Geometrical Compositing}

Datasets. For training the Appearance Compositing network, we use the COCO (Lin et al. 2014) dataset due to its large variety of natural images, containing both humans and associated ground truth segmentation masks. For the Geometric Compositing pipeline, we sample various backgrounds from the NYU Depth Dataset V2 (Nathan Silberman and Fergus 2012). This dataset contains 1, 449 RGB images, depicting 464 indoor scenes captured with a Kinect sensor. For each image, the dataset provides corresponding semantic annotations as well as aligned depth images. We consider the semantic classes "floor", "rug", "floor mat" and "yoga mat" in order to infer plausible support surfaces.

Quantitative Evaluation. Table 2 shows the performance of our Appearance Compositing network on images from the COCO validation dataset under the LPIPS metric. We first report the LPIPS metric between the initial images and their randomly perturbed versions (0.0971). Then we show how this error score is considerably improved when we apply our Appearance Compositing network, that uses VGG and L1 loss (from 0.0971 to 0.0588 ). Note that there is also a considerable reduction in the standard deviation. The re- 

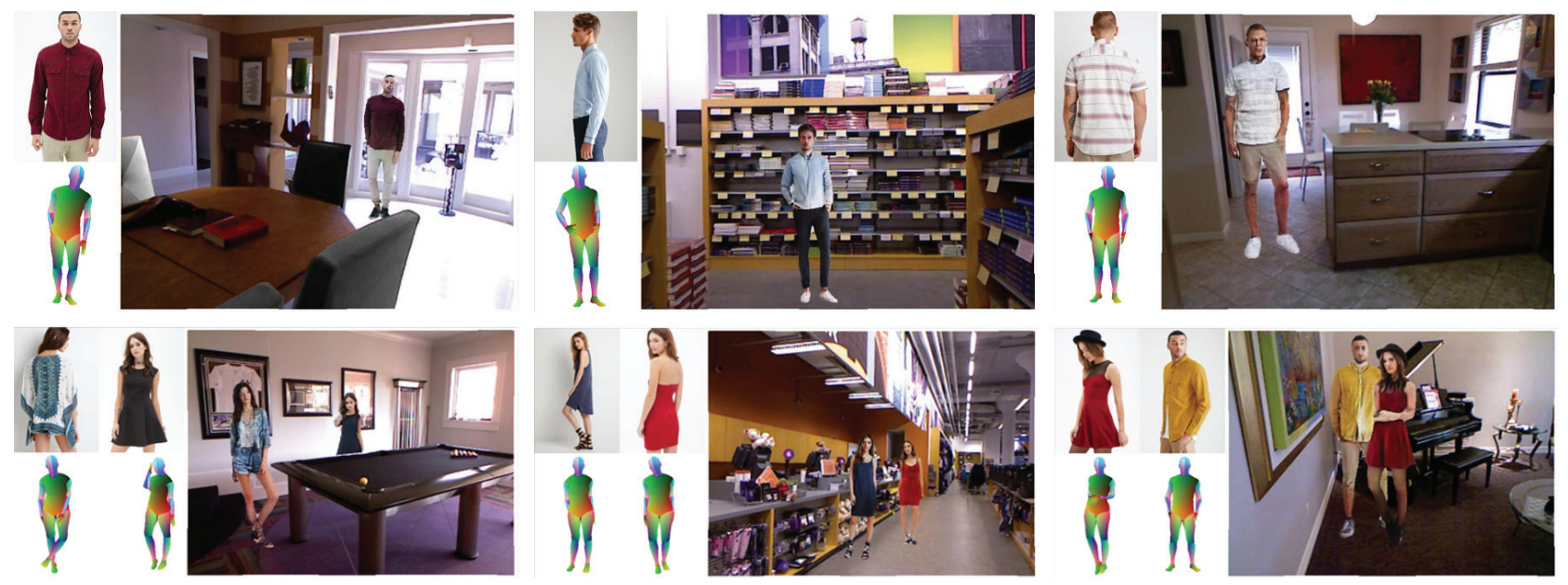

Figure 8: Sample images generated by our framework. For each example, we show the source image, the target 3d body mesh, and a scene with a geometrically plausible placement of the synthesized person. Please note that our framework allows for a positioning behind various objects, and the insertion of multiple people without breaking any geometrical scene properties.

sults further improve if we add a GAN loss to our network (from 0.0588 to 0.0542 ).

\begin{tabular}{|l|c|c|}
\hline & \multicolumn{2}{|c|}{ LPIPS score } \\
\hline Reference & Full image $\downarrow$ & Foreground $\downarrow$ \\
\hline \hline Perturbed & $0.0971 \pm 0.0596$ & $0.2569 \pm 0.0811$ \\
\hline Refined (L1+VGG loss) & $0.0588 \pm 0.0267$ & $0.1317 \pm 0.0514$ \\
\hline Refined (L1+VGG+GAN loss) & $\mathbf{0 . 0 5 4 2} \pm \mathbf{0 . 0 2 4 3}$ & $\mathbf{0 . 1 1 6 5} \pm \mathbf{0 . 0 4 8 0}$ \\
\hline
\end{tabular}

Table 2: LPIPS (smaller the better) on 1000 images from Microsoft COCO validation dataset.

In table 3 we report the Inception Score for 1000 composited images of humans on diverse backgrounds, generated by HUSC: with only the Geometrical Compositing, and when the Appearance Compositing module is also added. The score is improved when correcting the appearance of the geometrical composites, which indicate that the images become more realistic. As a comparison baseline, we checked the IS of the background images alone. Note that there is only a less than $10 \%$ drop, in the score of the final composite image, as compared to the original (real), background image.

\begin{tabular}{|l|c|}
\hline Image set & Inception Score $\uparrow$ \\
\hline \hline NYU Depth Dataset V2 & 6.96 \\
\hline HUSC (Only Geometrical Compositing) & 6.23 \\
\hline HUSC & 6.33 \\
\hline
\end{tabular}

Table 3: IS for images generated by our method with and without appearance compositing. We also show IS for the original background images selected from the NYU dataset.

Qualitative Examples. In fig. 8 we show examples of images generated by our proposed HUSC framework. For each example, we show the source image, the dense pose associated with the target body model and the resulting synthesized scene. Notice that the persons are naturally blended in the background scene at both geometrical and appearance levels. In fig. 7 we illustrate before and after results for our appearance compositing network. Our method adapts the foreground to the ambient scene context by darkening the appearance, and smooths the boundaries.

\section{Conclusions}

We have presented a HUman Synthesis and Scene Compositing framework (HUSC) for the realistic and controllable synthesis of humans with different appearance, in novel poses and scenes. By operating in the $3 \mathrm{~d}$ scene space rather than image space, except for late global image adaptation stages, and by taking into account scene semantics, we are able to realistically place the human impostor on support surfaces, handle scene scales and model occlusion. Moreover, by working with parametric $3 \mathrm{~d}$ human models and dense geometric correspondences, we can better control and localize the appearance transfer process during synthesis. The model produces pleasing qualitative results and obtains superior quantitative results on the DeepFashion dataset, making it practically applicable for photo editing, fashion virtual try-on, or for realistic data augmentation used for training large scale $3 \mathrm{~d}$ human sensing models.

Acknowledgments: This work was supported in part by the European Research Council Consolidator grant SEED, CNCS-UEFISCDI (PN-III-P4-ID-PCE-2016-0535, PN-IIIP4-ID-PCCF-2016-0180), the EU Horizon 2020 grant DEENIGMA (688835), and SSF.

\section{References}

Alhaija, H.; Mustikovela, S.; Geiger, A.; and Rother, C. 2018. Geometric image synthesis. In $(A C C V)$.

Alp Guler, R.; Neverova, N.; and Kokkinos, I. 2018. Densepose: Dense human pose estimation in the wild. In (CVPR). 
Balakrishnan, G.; Zhao, A.; Dalca, A. V.; Durand, F.; and Guttag, J. 2018. Synthesizing images of humans in unseen poses. In $(C V P R)$.

Dong, H.; Liang, X.; Gong, K.; Lai, H.; Zhu, J.; and Yin, J. 2018. Soft-gated warping-gan for pose-guided person image synthesis. In (NeurIPS), 474-484.

Dvornik, N.; Mairal, J.; and Schmid, C. 2018. Modeling visual context is key to augmenting object detection datasets. In (ECCV), 364-380.

Dwibedi, D.; Misra, I.; and Hebert, M. 2017. Cut, paste and learn: Surprisingly easy synthesis for instance detection. In (CVPR), 1301-1310.

Esser, P.; Sutter, E.; and Ommer, B. 2018. A variational u-net for conditional appearance and shape generation. In (CVPR).

Gong, K.; Liang, X.; Li, Y.; Chen, Y.; Yang, M.; and Lin, L. 2018. Instance-level human parsing via part grouping network. In (ECCV).

Goodfellow, I.; Pouget-Abadie, J.; Mirza, M.; Xu, B.; Warde-Farley, D.; Ozair, S.; Courville, A.; and Bengio, Y. 2014. Generative adversarial nets. In (NIPS).

Grigorev, A.; Sevastopolsky, A.; Vakhitov, A. T.; and Lempitsky, V. S. 2018. Coordinate-based texture inpainting for pose-guided image generation. CoRR abs/1811.11459.

Han, X.; Wu, Z.; Wu, Z.; Yu, R.; and Davis, L. S. 2018. Viton: An image-based virtual try-on network. In (CVPR).

Isola, P.; Zhu, J.-Y.; Zhou, T.; and Efros, A. A. 2017. Imageto-image translation with conditional adversarial networks. In (CVPR).

Johnson, J.; Alahi, A.; and Fei-Fei, L. 2016. Perceptual losses for real-time style transfer and super-resolution. In $(E C C V)$.

Lassner, C.; Pons-Moll, G.; and Gehler, P. V. 2017. A generative model of people in clothing. In (ICCV), 853-862.

Lee, H.-Y.; Tseng, H.-Y.; Huang, J.-B.; Singh, M. K.; and Yang, M.-H. (2018). Diverse image-to-image translation via disentangled representations. In $(E C C V)$.

Li, Y.; Huang, C.; and Loy, C. C. 2019. Dense intrinsic appearance flow for human pose transfer. In (CVPR).

Lin, T.-Y.; Maire, M.; Belongie, S.; Hays, J.; Perona, P.; Ramanan, D.; Dollár, P.; and Zitnick, C. L. 2014. Microsoft coco: Common objects in context. In $(E C C V)$.

Liu, Z.; Luo, P.; Qiu, S.; Wang, X.; and Tang, X. 2016. Deepfashion: Powering robust clothes recognition and retrieval with rich annotations. In $(C V P R)$.

Loper, M.; Mahmood, N.; Romero, J.; Pons-Moll, G.; and Black, M. J. 2015. Smpl: A skinned multi-person linear model. ACM transactions on graphics (TOG) 34(6):248.

Luan, F.; Paris, S.; Shechtman, E.; and Bala, K. 2017. Deep photo style transfer. In (CVPR), 4990-4998.

Ma, L.; Jia, X.; Sun, Q.; Schiele, B.; Tuytelaars, T.; and Van Gool, L. 2017. Pose guided person image generation. In (NIPS), 406-416.
Ma, L.; Sun, Q.; Georgoulis, S.; Van Gool, L.; Schiele, B.; and Fritz, M. 2018. Disentangled person image generation. In (CVPR), 99-108.

Nathan Silberman, Derek Hoiem, P. K., and Fergus, R. 2012. Indoor segmentation and support inference from rgbd images. In $(E C C V)$.

Neverova, N.; Alp Guler, R.; and Kokkinos, I. 2018. Dense pose transfer. In (ECCV), 123-138.

Nguyen, A.; Clune, J.; Bengio, Y.; Dosovitskiy, A.; and Yosinski, J. 2017. Plug \& play generative networks: Conditional iterative generation of images in latent space. In (CVPR), 4467-4477.

Pumarola, A.; Agudo, A.; Sanfeliu, A.; and Moreno-Noguer, F. 2018. Unsupervised person image synthesis in arbitrary poses. $(C V P R)$.

Remez, T.; Huang, J.; and Brown, M. 2018. Learning to segment via cut-and-paste. In (ECCV), 37-52.

Salimans, T.; Goodfellow, I.; Zaremba, W.; Cheung, V.; Radford, A.; and Chen, X. 2016. Improved techniques for training gans. In (NIPS), 2234-2242.

Siarohin, A.; Sangineto, E.; Lathuilière, S.; and Sebe, N. 2018. Deformable gans for pose-based human image generation. In $(C V P R)$.

Tsai, Y.-H.; Shen, X.; Lin, Z.; Sunkavalli, K.; Lu, X.; and Yang, M.-H. 2017. Deep image harmonization. In (CVPR).

Varol, G.; Romero, J.; Martin, X.; Mahmood, N.; Black, M. J.; Laptev, I.; and Schmid, C. 2017. Learning from synthetic humans. In (CVPR).

Wang, Z.; Bovik, A. C.; Sheikh, H. R.; and Simoncelli, E. P. 2004. Image quality assessment: From error measurement to structural similarity. (TIP).

Wang, T.-C.; Liu, M.-Y.; Zhu, J.-Y.; Tao, A.; Kautz, J.; and Catanzaro, B. 2018. High-resolution image synthesis and semantic manipulation with conditional gans. In (CVPR).

Yi, Z.; Zhang, H. R.; Tan, P.; and Gong, M. 2017. Dualgan: Unsupervised dual learning for image-to-image translation. In (ICCV), 2868-2876.

Zanfir, A.; Marinoiu, E.; Zanfir, M.; Popa, A.-I.; and Sminchisescu, C. 2018. Deep network for the integrated 3d sensing of multiple people in natural images. In (NIPS).

Zanfir, A.; Marinoiu, E.; and Sminchisescu, C. 2018. Monocular 3D Pose and Shape Estimation of Multiple People in Natural Scenes - The Importance of Multiple Scene Constraints. In $(C V P R)$.

Zanfir, M.; Popa, A. I.; and Sminchisescu, C. 2018. Human appearance transfer. In $(C V P R)$.

Zhang, R.; Isola, P.; Efros, A. A.; Shechtman, E.; and Wang, O. 2018. The unreasonable effectiveness of deep features as a perceptual metric. In $(C V P R)$. 